\title{
A two-agent model applied to the biological control of the sugarcane borer (Diatraea saccharalis) by the egg parasitoid Trichogramma galloi and the larvae parasitoid Cotesia flavipes
}

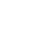

Sándor Molnár ${ }^{1}$, Inmaculada López ${ }^{2^{*}}$, Manuel Gámez ${ }^{2^{*}}$, József Garay ${ }^{3}$

${ }^{1}$ Szent István University/Hungary, ${ }^{2}$ University of Almeria/Spain, ${ }^{3}$ MTA-ELTE Theoretical

Biology and Evolutionary Ecology Research Group and Department of Plant Systematics,

Ecology and Theoretical Biology, L. Eötvös University/Hungary (*Corresponding author:

Department of Mathematics, University of Almeria, 04120, Spain, Phone: +34 950015667, E-

mail:mgamez@ual.es)

\begin{abstract}
The paper is aimed at a methodological development in biological pest control. The considered one pest two-agent system is modelled as a verticum-type system. Originally, linear verticum-type systems were introduced by one of the authors for modelling certain industrial systems. These systems are hierarchically composed of linear subsystems such that a part of the state variables of each subsystem affect the dynamics of the next subsystem. Recently, verticum-type system models have been applied to population ecology as well, which required the extension of the concept a verticum-type system to the nonlinear case.
\end{abstract}

In the present paper the general concepts and technics of nonlinear verticum-type control systems are used to obtain biological control strategies in a two-agent system. For the illustration of this verticum-type control, these tools of mathematical systems theory are applied to a dynamic model of interactions between the egg and larvae populations of the sugarcane borer (Diatraea saccharalis) and its parasitoids: the egg parasitoid Trichogramma galloi and the larvae parasitoid Cotesia flavipes.

In this application a key role is played by the concept of controllability, which means that it is possible to steer the system to an equilibrium in given time. In addition to a usual linearization, the basic idea is a decomposition of the control of the whole system into the control of the subsystems, making use of the verticum structure of the population system. The main aim of this study is to show several advantages of the verticum (or decomposition) approach over the classical control theoretical model (without decomposition). For example, in the case of verticum control the pest larval density decreases below the critical threshold value much quicker than without decomposition. Furthermore, it is also shown that the verticum approach may be better even in terms of cost effectiveness. The presented optimal control methodology also turned out to be an efficient tool for the "in silico" analysis of the cost-effectiveness of different biocontrol strategies, e.g. by answering the question how far it is cost-effective to speed up the reduction of the pest larvae density, or along which trajectory this reduction should be carried out.

Keywords: biological pest control, verticum-type system, controllability

\section{INTRODUCTION}

Apart from the cultivation of sugar cane for traditional uses, over the last decades, its application as renewable energy source in ethanol production increased the interest in its production in tropical and subtropical areas. As pointed out by Rafikov and Silveira (2014), large-scale, monocultural farming and long crop duration offer certain pests good conditions to establish. In particular, sugarcane borer Diatraea sacharalis excavating galleries inside the sugarcane plants, is the most important pest in South-East Brazil damaging sugarcane crops. 
For the detailed description of the damage see e.g. Macedo and Botelho (1988) and Parra et al. (2002). Nowadays, based on environmental considerations, biological pest control (shortly biocontrol) in most cases is preferable to the chemical one. In biocontrol the density of the pest species is reduced by the release of natural enemies (mostly predators or parasitoids) as control agents. Once we have a dynamic model for the description of the interaction between pest and agent(s), Control Theory, or more generally, Mathematical Systems Theory (MST) offer adequate tools for the analysis of and design of biocontrol activity.

8 Optimal control methodology have been used e.g. for the malaria vector control by the release 9 of transgenic mosquitoes in Rafikov et al. (2009). Basic concepts of MST such as controllability (i.e. steering a system to an equilibrium) and observability (monitoring the whole system by observing only some components of it) have been first applied to frequency-dependent population genetic systems by Varga $(1989,1992)$, followed by evolutionary models of Scarelli and Varga (2002) and López et al. (2004). MST methodology has been also applied to densitydependent population systems in Varga et al. (2002, 2003), Shamandy (2005), Varga (2008a), López et al. (2007a, b), Gámez et al. (2009), and to spatially structured population models in Gámez et al. (2011, 2012). For reviews on the topic we refer the reader to Varga (2008b), Gámez (2011) and Varga et al. (2013).

In the pest control methodology developed in the present paper, the particular verticum-type structure of the dynamical control model plays a key role. Linear verticum-type systems were introduced by Molnár (1989) for modelling certain industrial systems. Such systems are hierarchically composed of subsystems such that a part of the state variables of each subsystem affect the dynamics of the next subsystem. Systems-theoretical properties of such systems were studied in Molnár (1993), Molnár and Szigeti (1994), Gámez et al. (2010), and for the monitoring problem of nonlinear verticum-type population systems see Molnár et al. (2012).

The main aim of the present is to show several advantages of the verticum (or decomposition) approach (method a)) over the classical control theoretical model (without decomposition, method b)). First, In case a) pest larval density decreases below the threshold value much quicker than in case b), saving in this way the major part of the crop. For example, in the case a) the pest larval density decreases below the critical threshold value much quicker than in case b). Furthermore, it is also shown that the approach a) may be better even in terms of cost effectiveness. The presented optimal control methodology also turned out to be an efficient tool for the "in silico" analysis of the cost-effectiveness of different biocontrol strategies, e.g. by answering the question how far it is cost-effective to speed up the reduction of the pest larvae density, or along which trajectory this reduction should be carried out.

The paper is organized as follows. In the Material and Methods Section, some basics of classical systems theory are shortly summarized, and the concept of a nonlinear verticum-type system and a sufficient condition for local controllability to equilibrium are recalled. The Results Section is dedicated to the application of these mathematical tools to a two-agent biocontrol model describing interactions between the egg and larvae populations of the sugarcane borer (Diatraea saccharalis) and its parasitoids: the egg parasitoid Trichogramma galloi and the larvae parasitoid Cotesia flavipes, based on the model of Rafikov and Silveira (2013). We find a biological control strategy to steer the population to a new desired equilibrium, where the pest larval density is below the economic damage level. Furthermore, we also show how to control the system to the given equilibrium, along a given trajectory for the pest larval density, and the costs of different control strategies are also analyzed in a numerical illustration. Finally, a Discussion Section closes the paper. 
2 Mathematical tools: concept of controllability

3 Given $n, r \in \mathbf{N}$, let $F: \mathbf{R}^{n} \times \mathbf{R}^{r} \rightarrow \mathbf{R}^{n}$ be a continuously differentiable function. For a 4 reference control value $u^{*} \in \mathbf{R}^{r}$, let $x^{*} \in \mathbf{R}^{n}$ be such that $F\left(x^{*}, u^{*}\right)=0$. Let us fix a time 5 interval $[0, T]$, and for each $\varepsilon>0$, consider the set $U_{\varepsilon}[0, T]$ of $\varepsilon$-small controls on $[0, T]$. 6 (For the technical details see Lee and Markus, 1971). From the latter reference we recall the 7 following statement on the existence and uniqueness of a solution for small controls:

8 There exists $\varepsilon_{0} \in \mathbf{R}^{+}$such that for all $u \in U_{\varepsilon_{0}}[0, T]$ and $x^{0} \in \mathbf{R}^{n}$ with $\left|x^{0}-x^{*}\right|<\varepsilon_{0}$ the initial 9 value problem

$$
\begin{aligned}
& \dot{x}(t)=F\left(x(t), u^{*}+u(t)\right) \quad(t \in[0, T]) \\
& x(0)=x^{0}
\end{aligned}
$$

has a unique solution. We notice that $x^{*}$ is an equilibrium state for the zero-control system.

Control system (1.1)-(1.2) is said to be locally controllable to $x^{*}$ on $[0, T]$, if there exists $0<\varepsilon<\varepsilon_{0}$ such that for all $x^{0}$ from the $\varepsilon$-neighbourhood of $x^{*}$, there is a control $u \in U_{\varepsilon}[0, T]$ that controls the initial state $x^{0}$ to equilibrium $x^{*}$, i.e. for the solution $x$ of the initial value problem (1.1)-(1.2), equality $x(T)=x^{*}$ holds.

Let us linearize system (1.1)-(1.2) around $\left(x^{*}, u^{*}\right)$, introducing the corresponding Jacobians

$$
A:=\frac{\partial}{\partial x} F\left(x^{*}, u^{*}\right), \quad B:=\frac{\partial}{\partial u} F\left(x^{*}, u^{*}\right) .
$$

From Lee and Markus (1971) we recall the following sufficient condition for local controllability:

If

$$
\operatorname{rank}\left[\begin{array}{c}
B \\
A B \\
\vdots \\
A^{n-1} B
\end{array}\right]=n
$$

then system (1.1)-(1.2) is locally controllable to $x^{*}$ on $[0, T]$.

\section{Verticum-structured model}

In this section we introduce some notations and, from Molnár et al. (2013), we recall the definition of a nonlinear verticum-type control system and some results to be applied in the present paper.

Given $\quad k, n_{i}, r_{i} \in N(i=0, \ldots, k), n:=\sum_{i=0}^{k} n_{i}, r:=\sum_{i=0}^{k} r_{i} ; \quad$ let $\quad F: \mathbf{R}^{n} \times \mathbf{R}^{r} \rightarrow \mathbf{R}^{n} \quad$ be $\quad$ a

continuously differentiable function. 
1 For a constant reference control $u^{*}:=\left(u_{0}^{*}, u_{1}^{*}, \ldots, u_{k}^{*}\right) \in \mathbf{R}^{r}$ with $u_{i}^{*} \in \mathbf{R}^{r_{i}}(i=0, \ldots, k)$, let $2 \quad x^{*}:=\left(x_{0}^{*}, x_{1}^{*}, \ldots, x_{k}^{*}\right) \in \mathbf{R}^{n}$ with $x_{i}^{*} \in \mathbf{R}^{n_{i}}(i=0, \ldots, k)$ such that $F\left(x^{*}, u^{*}\right)=0$.

3 Let us fix a time interval $[0, T]$, and for each $\varepsilon>0$, let $U_{\varepsilon}[0, T]$ be the class of $\varepsilon$-small 4 controls on $[0, T]$.

5 Consider the nonlinear control system

6

7 and for all $i=1, \ldots, k$,

and define

Definition have $F\left(x^{*}, u^{*}\right)=0$. value problem

where

$$
\dot{x}_{0}=F_{0}\left(x_{0}, u_{0}^{*}+u_{0}\right) ; \quad F_{0}: \mathbf{R}^{n_{0}} \times \mathbf{R}^{r_{0}} \rightarrow \mathbf{R}^{n_{0}},
$$

$$
\dot{x}_{i}=F_{i}\left(x_{i}, x_{i-1}, u_{i}^{*}+u_{i}\right) ; \quad F_{i}: \mathbf{R}^{n_{i}} \times \mathbf{R}^{n_{i-1}} \times \mathbf{R}^{r_{i}} \rightarrow \mathbf{R}^{n_{i}},
$$

$$
F\left(x, u^{*}+u\right):=\left(F_{0}\left(x_{0}, u_{0}^{*}+u_{0}\right), F_{1}\left(x_{1}, x_{0}, u_{1}^{*}+u_{1}\right), \ldots, F_{k}\left(x_{k}, x_{k-1}, u_{k}^{*}+u_{k}\right)\right)
$$

$$
\dot{x}=F\left(x, u^{*}+u\right)
$$

is said to be a (nonlinear) verticum-type control system with subsystems $\left(V_{i}\right)(i=0, \ldots, k)$.

We note that $x^{*}$ fixed above, is an equilibrium of the zero-dynamics of (V), i.e., for $u=0$ we

Remark 1. Equations $\left(V_{i}\right)$ do not define a standard control system in this setting, because of the presence of the "exogenous" variable $x_{i-1}$ connecting it to equation $\left(V_{i-1}\right)(i=1, \ldots, k)$. Nevertheless, $\left(V_{i}\right)$ are also called subsystems of system (V).

Remark 2. From the existence and uniqueness of the solution of (1.1)-(1.2), we obtain that there exists $\varepsilon_{0}>0$ such that for all $u \in U_{\varepsilon_{0}}[0, T]$ and $x^{0} \in \mathbf{R}^{n}$ with $\left|x^{0}-x^{*}\right|<\varepsilon_{0}$ the initial

$$
\begin{aligned}
& \dot{x}(t)=F\left(x(t), u^{*}+u(t)\right) \quad(\text { for a.e. } t \in[0, T]) \\
& x(0)=x^{0}
\end{aligned}
$$

has a unique solution. In what follows $T>0$ will be fixed and concerning controllability, the reference to interval $[0, T]$ will be often suppressed.

To study controllability of system $(V)$, let us linearize system $\left(V_{0}\right)$, at equilibrium $\left(x_{0}^{*}, u_{0}^{*}\right)$, obtaining the linearized systems

$$
\dot{x}_{0}=A_{00} x_{0}+B_{0} u_{0},
$$

$$
A_{00}=\frac{\partial F_{0}}{\partial x_{0}}\left(x_{0}^{*}, u_{0}^{*}\right), \quad B_{0}=\frac{\partial F_{0}}{\partial u_{0}}\left(x_{0}^{*}, u_{0}^{*}\right)
$$


1 and for all $(i=1, \ldots, k)$, substituting $x_{i-1}$ in $\left(V_{i}\right)$ with its equilibrium value $x_{i-1}^{*}$, we similarly 2 linearize $\left(V_{i}\right)$ with respect to variables $\left(x_{i}, u_{i}\right)$, at the corresponding equilibrium $\left(x_{i}^{*}, u_{i}^{*}\right)$, 3 obtaining the linearized systems

$$
\dot{x}_{i}=A_{i i} x_{i}+B_{i} u_{i}
$$

$$
\text { with } A_{i i}=\frac{\partial F_{i}}{\partial x_{i}}\left(x_{i}^{*}, x_{i-1}^{*}, u_{i}^{*}\right) ; \quad B_{i}=\frac{\partial F_{i}}{\partial u_{i}}\left(x_{i}^{*}, x_{i-1}^{*}, u_{i}^{*}\right) \quad(i=1, \ldots, k) \text {. }
$$

Then from Molnár et al. (2013) we have the following sufficient condition for local controllability of nonlinear verticum-type systems:

8 Theorem 1. (Molnár et al., 2013). If

$$
\operatorname{rank}\left[\begin{array}{c}
B_{i} \\
A_{i i} B_{i} \\
\vdots \\
A_{i i}^{n_{i}-1} B_{i}
\end{array}\right]=n_{i} \quad(i=0, \ldots, k),
$$

then control system $(V)$ is locally controllable to equilibrium $x^{*}$. Local controllability means that from nearby states the system can be controlled to $x^{*}$ using small controls. Intuitively, the above theorem says that the problem of controllability of the whole system can be decomposed into controllability problems concerning the subsystems.

In what follows the above result will be applied to the analysis of a control system modeling pest control.

\section{Two-agent biological control model}

For the mathematical model we will use to describe the interactions between the sugarcane borer (Diatraea saccharalis) and its egg parasitoid (Trichogramma galloi) and larvae parasitoid (Cotesia flavipes), the following parameters are needed:

$r$ is the intrinsic oviposition rate of female sugarcane borer;

$K$ is the potential maximum of oviposition rate of female sugarcane borer;

$m_{1}, m_{2}, m_{3}$ and $m_{4}$ are the mortality rates of the egg, egg parasitoid, larvae and larvae parasitoid populations, respectively;

$n_{1}$ is the fraction of the sugarcane borer larvae population which emerges from the eggs in unit time;

$n_{3}$ is the fraction of the un-parasitized sugarcane borer larvae from which pupae emerge in unit time;

$\alpha$ and $\beta$ are the intrinsic parasitism rate of the egg and larvae parasitoids, respectively;

$\gamma_{1}$ and $\gamma_{2}$ are the survival rates of parasitized eggs and larvae to adult age, respectively

In Molnar et al. (2013) a model for the integrated pest control of sugarcane borer was considered, where the release of a single agent was combined with the application of a pesticide. Now we consider the case of a purely biological control with two agents, based on the mathematical model of interactions between the sugarcane borer and its egg and larvae parasitoids studied by Rafikov and Silveira (2013): 


$$
\begin{aligned}
\frac{d x_{1}}{d t} & =r x_{1}\left(1-\frac{x_{1}}{K}\right)-m_{1} x_{1}-n_{1} x_{1}-\alpha x_{1} x_{2} \\
\frac{d x_{2}}{d t} & =\alpha \gamma_{1} x_{1} x_{2}-m_{2} x_{2} \\
\frac{d x_{3}}{d t} & =n_{1} x_{1}-m_{3} x_{3}-n_{3} x_{3}-\beta x_{3} x_{4} \\
\frac{d x_{4}}{d t} & =\beta \gamma_{2} x_{3} x_{4}-m_{4} x_{4},
\end{aligned}
$$

where $x_{1}$ is the un-parazitized egg population density of the sugarcane borer, $x_{2}$ the density of the adult egg parasitoid Trichogramma galloi, $x_{3}$ the un-parazitized larvae density of the sugarcane borer, $x_{4}$ the density of the adult larvae parasitoid Cotesia flavipes, and the interpretation of the model parameters (coefficients) was given at the beginning of this section

In our study we will apply the following parameter set, resulting from field experiments (see Parra et al., 2002), also applied for modelling in Rafikov and Silveira (2013):

$r=0.19 ; K=25000 ; m_{1}=0.03566 ; m_{2}=0.03566 ; m_{3}=0.00256 ; m_{4}=1 ; n_{1}=0.1$; $n_{3}=0.02439 ; \alpha=0.0000075 ; \beta=0.0000083 ; \gamma_{1}=2.29 ; \gamma_{2}=40$.

The units of all parameters are defined from the requirement that each term on the right-hand sides of the equations (3.1) must have dimension density/unit time.

Remark 3. Our model (3.1a)-(3.1.d) is not a compartment type model, and is not intended to include all development stages of all involved species, it can rather be considered as two "hostparasitoid" models linked together through transformations of pest eggs into egg parasitoid adults (pest larvae into larvae parasitoid adults). Our aim was to build up a minimal model, sufficient for the optimization of the biological control of the sugar cane borer. We emphasize that for our purpose it is enough to take account of the parasitized eggs (and parasitized larvae) in the last negative term of equation (3.1a) (and 3.1c)).

There may be different ways to simplify the modelling of the mechanism of parazitation. The model of Rafikov and Limeira (2012) consideres that the adult egg-parasitoids only infect eggs at the beginning of the adult's life. Our model instead, allows all the adult parasitoids to infect eggs, not just the adults as they emerge, which is experimentally supported, see e.g. Cabello and Vargas (1988) and Nogueira and Parra (1994), although it is also true that, Trichogramma females have higher fecundity in the the first couple of days of their adult stage. In any case, our hypothesis, as a possible modelling approximation (by constant average infection rates $\alpha$ and $\beta$ ) is also justified.

We also note that $-n_{3} x_{3}$ is the part of un-parasitized pest larvae that leave the larval stage by development, and it does not enter in any other equation of our model.

In Rafikov and Silveira (2013) five equilibrium points of model (3.1) were obtained and also the corresponding stability analysis was carried out. We are interested in the only strictly positive equilibrium point $x^{*}=\left(x_{1}^{*}, x_{2}^{*}, x_{3}^{*}, x_{4}^{*}\right)$, where 


$$
\begin{aligned}
& x_{1}^{*}=\frac{m_{2}}{\alpha \gamma_{1}}, \\
& x_{2}^{*}=\frac{1}{\alpha}\left[r\left(1-\frac{m_{2}}{\alpha \gamma_{1} K}\right)-m_{1}-n_{1}\right], \\
& x_{3}^{*}=\frac{m_{4}}{\beta \gamma_{2}}, \\
& x_{4}^{*}=\frac{n_{1} m_{2} \gamma_{2}}{\alpha \gamma_{1} m_{4}}-\frac{m_{3}+n_{3}}{\beta} .
\end{aligned}
$$

2 From now on, for model (3.1) in the subsequent sections we will consider this equilibrium 3 denoted by $x^{*}$. In Rafikov and Silveira (2013), using linearization, it was obtained that 4 conditions

$$
r>m_{1}+n_{1}, \quad \alpha>\frac{r m_{2}}{\gamma_{1} K\left(r-m_{1}-n_{1}\right)} \quad \text { and } \quad \beta>\frac{\alpha \gamma_{1}\left(m_{3}+n_{3}\right) m_{4}}{\gamma_{2} n_{1} m_{2}}
$$

imply not only strict positivity of $x^{*}$, but also its asymptotic stability. In biological terms, the latter means stable coexistence of the population system.

\section{RESULTS}

\section{Controlling to a desired equilibrium}

In case of a strong pest invasion, it may happen that the sugarcane borer larval density tends to a too high equilibrium value that causes a serious damage in the crop. Then, it is appropriate to apply natural enemies in order to control the system to a new equilibrium state where pest larval density is below the economically determined threshold, in a given time $T$.

In our control model, we shall consider $\varepsilon$-small controls introduced in the previous section. For system (3.1), we set up a general optimal control problem with control dynamics

$$
x^{\prime}=F\left(x_{1}, x_{2}, x_{3}, x_{4}, u\right):=\left[\begin{array}{l}
r x_{1}\left(1-\frac{x_{1}}{K}\right)-m_{1} x_{1}-n_{1} x_{1}-\alpha x_{1} x_{2} \\
\alpha \gamma_{1} x_{1} x_{2}-m_{2} x_{2}+U_{2} \\
n_{1} x_{1}-m_{3} x_{3}-n_{3} x_{3}-\beta x_{3} x_{4} \\
\beta \gamma_{2} x_{3} x_{4}-m_{4} x_{4}+U_{4}
\end{array}\right],
$$

where functions $U_{2}$ and $U_{4}$ describe the time-dependent rate of release of egg and larvae parasitoids, respectively, realizing the biological control of the pest.

Our purpose is to control system (3.1) into a required new equilibrium state, applying biological control. In Rafikov et al. (2008), an optimal control technique was developed where a feedback asymptotically controls the system into a desired equilibrium, see also Rafikov and Limeira, (2012). We point out that in our optimal control models our aim will be to determine a corresponding pest control strategy that steers the system to $x_{d}^{*}$ in a given time $T$. 
1 Let $x_{d 3}^{*}$ be the target density of pest larvae fixed below the critical threshold value, to avoid

2 serious economic damages. Following the steps of Rafikov and Silveira (2013), we can obtain

3 the corresponding value of the desired positive equilibrium state $x_{d}^{*}=\left(x_{d 1}^{*}, x_{d 2}^{*}, x_{d 3}^{*}, x_{d 4}^{*}\right)$

4 with control $u^{*}=\left(u_{2}^{*}, u_{4}^{*}\right)$ that satisfies the following system of equations:

5

$$
\begin{aligned}
& r x_{1}^{*}\left(1-\frac{x_{1}^{*}}{K}\right)-m_{1} x_{1}^{*}-n_{1} x_{1}^{*}-\alpha x_{1}^{*} x_{2}^{*}=0 \\
& \alpha \gamma_{1} x_{1}^{*} x_{2}^{*}-m_{2} x_{2}^{*}+u_{2}^{*}=0 \\
& n_{1} x_{1}^{*}-m_{3} x_{3}^{*}-n_{3} x_{3}^{*}-\beta x_{3}^{*} x_{4}^{*}=0 \\
& \beta \gamma_{2} x_{3}^{*} x_{4}^{*}-m_{4} x_{4}^{*}+u_{4}^{*}=0 .
\end{aligned}
$$

6 In this system, in addition to the pest larval density, $x_{3}^{*}=x_{d 3}^{*}$, we can also fix a desired pest

7 eggs density $x_{1}^{*}=x_{d 1}^{*}$, and then solve system (3.5) for the remaining four unknowns to obtain

$$
\begin{gathered}
x_{d 2}^{*}=\frac{1}{\alpha}\left[r\left(1-\frac{x_{d 1}^{*}}{K}\right)-m_{1}-n_{1}\right], \\
x_{d 4}^{*}=\frac{n_{1} x_{d 1}^{*}-x_{d 3}^{*}\left(m_{3}+n_{3}\right)}{\beta x_{d 3}^{*}} ;
\end{gathered}
$$

and for the control variables $u^{*}=\left(u_{2}^{*}, u_{4}^{*}\right)$,

$$
u_{2}^{*}=m_{2} x_{d 2}^{*}-\alpha \gamma_{1} x_{d 1}^{*} x_{d 2}^{*}, \quad u_{4}^{*}=m_{4} x_{d 4}^{*}-\beta \gamma_{2} x_{d 3}^{*} x_{d 4}^{*} .
$$

The positivity of these $u_{2}^{*}, u_{4}^{*}$ indicates if the desired equilibrium can be maintained by constant agent releases. Hence the desired equilibrium is

$$
x_{d}^{*}=\left(x_{d 1}^{*}, \frac{1}{\alpha}\left[r\left(1-\frac{x_{d 1}^{*}}{K}\right)-m_{1}-n_{1}\right], \quad x_{d 3}^{*}, \frac{n_{1} x_{d 1}^{*}-x_{d 3}^{*}\left(m_{3}+n_{3}\right)}{\beta x_{d 3}^{*}}\right) .
$$

This equilibrium will be positive, if the following conditions hold:

$$
r>m_{1}+n_{1}, \quad n_{1}>\frac{x_{d 3}^{*}\left(m_{3}+n_{3}\right)}{x_{d 1}^{*}}, \quad \text { with } x_{d 1}^{*}, x_{d 3}^{*}>0 .
$$

The positivity of $u_{2}^{*}, u_{4}^{*}$ in (3.6) indicates if the desired equilibrium can be maintained by constant agent releases. Moreover, from Rafikov and Silveira (2013) we know that the above positive equilibrium is asymptotically stable, therefore, the populations coexist in a common environment.

Control system (3.4) takes the form

$$
\dot{x}=F\left(x, u^{*}+u(t)\right),
$$


1 Here, for $U(t)$ of (3.4) we have $U(t)=u^{*}+u(t)$ where $u(t)=\left(u_{2}(t), u_{4}(t)\right)$ with $2 \quad\left|u_{2}(t)\right| \leq u_{2}^{*}$ and $\left|u_{4}(t)\right| \leq u_{4}^{*} \quad(t \in[0, T])$. Obviously $u(t)$ can be negative but $u^{*}+u(t)$ 3 remains non negative.

4 Given a desired equilibrium $x_{d}^{*}$ obtained from (3.7), to constant controls $u^{*}$ defined in (3.6) 5 and with $u(t):=0 \quad(t \in[0, T])$, there corresponds this equilibrium $x_{d}^{*}$.

6 From now on, for model (3.9) we shall use $u^{*}$ as it is defined in (3.6) and $u^{*}+u(t)$ is 7 interpreted as the total release of parasitoids at time $t$.

8 Below we show that system (3.9) is locally controllable to $x_{d}^{*}$ on $[0, T]$.

We note that from Remark 2 and from the continuous dependence of the solution on the ws that for controls small enough, the solutions of system (3.9) remain in the positive orthant.

Our main objective is a qualitative and quantitative analysis of control system (3.9), applying the theoretical results of the previous section, concerning nonlinear verticum-type control systems. In the present subsection, Theorem 1 will be applied to show that our population system can be controlled into equilibrium.

We start with the analysis of the first subsystem

$$
\frac{d x_{1}}{d t}=r x_{1}\left(1-\frac{x_{1}}{K}\right)-m_{1} x_{1}-n_{1} x_{1}-\alpha x_{1} x_{2}
$$

$$
\frac{d x_{2}}{d t}=\alpha \gamma_{1} x_{1} x_{2}-m_{2} x_{2}+u_{2}^{*}+u_{2} \text {. }
$$

With function $F_{0}: \mathbf{R}^{3} \rightarrow \mathbf{R}^{2}$

$$
F_{0}\left(x_{1}, x_{2}, u_{2}^{*}+u_{2}\right):=\left(\begin{array}{c}
r x_{1}\left(1-\frac{x_{1}}{K}\right)-m_{1} x_{1}-n_{1} x_{1}-\alpha x_{1} x_{2} \\
\alpha \gamma_{1} x_{1} x_{2}-m_{2} x_{2}+u_{2}^{*}+u_{2}
\end{array}\right),
$$

control system (3.10a) and (3.10b) takes the form

$$
\dot{x}^{1}=F_{0}\left(x^{1}, u_{2}^{*}+u_{2}(t)\right),
$$

where $x^{1}=\left(x_{1}, x_{2}\right)$ and $\left|u_{2}(t)\right| \leq u_{2}^{*}$. The latter is a requirement in order to $u_{2}^{*}+u_{2}(t)$ represents only "introduction" of egg parasitoid.

Obviously, to $u_{2}^{*}$ defined in (3.6) and $u_{2}(t):=0 \quad(t \in[0, T])$, there corresponds the desired positive equilibrium $x_{d}^{1^{*}}:=\left(x_{d 1}^{*}, x_{d 2}^{*}\right)$.

Now we show that control system (3.10) is locally controllable to $x_{d}^{1^{*}}$ on $[0, T]$. For the application of sufficient condition (1.3), let us calculate the linearization

$$
A_{00}:=\frac{\partial F_{0}}{\partial x^{1}}\left(x_{d}^{1^{*}}, u_{2}^{*}\right)=\left[\begin{array}{cc}
-\frac{r}{K} x_{d 1}^{*} & -\alpha x_{d 1}^{*} \\
\alpha \gamma_{1} x_{d 2}^{*} & 0
\end{array}\right], B_{0}:=\frac{\partial F_{0}}{\partial u_{2}}\left(x_{d}^{1^{*}}, u_{2}^{*}\right)=\left[\begin{array}{l}
0 \\
1
\end{array}\right] .
$$

Since 


$$
\operatorname{det}\left[B_{0} \mid A_{00} B_{0}\right]=\alpha \cdot x_{d 1}^{*} \neq 0
$$

2 we get $\operatorname{rank}\left[B_{0} \mid A_{00} B_{0}\right]=2$, and applying sufficient condition (1.3) we obtain local 3 controllability of system (3.10) into $x_{d}^{1^{*}}$ on interval $[0, T]$.

4 Analogously, let us consider the second subsystem

$$
\frac{d x_{3}}{d t}=n_{1} x_{1}-m_{3} x_{3}-n_{3} x_{3}-\beta x_{3} x_{4}
$$

$$
\frac{d x_{4}}{d t}=\beta \gamma_{2} x_{3} x_{4}-m_{4} x_{4}+u_{4}^{*}+u_{4} \text {. }
$$

$6 \quad$ With notation $F_{1}: R^{4} \rightarrow R^{2}$

$$
F_{1}\left(x_{1}, x_{3}, x_{4}, u_{4}^{*}+u_{4}\right):=\left(\begin{array}{c}
n_{1} x_{1}-m_{3} x_{3}-n_{3} x_{3}-\beta x_{3} x_{4} \\
\beta \gamma_{2} x_{3} x_{4}-m_{4} x_{4}+u_{4}^{*}+u_{4}
\end{array}\right),
$$

for control system (3.11a) and (3.11b) we get

$$
\dot{x}^{2}=F_{1}\left(x_{1}, x^{2}, u_{4}^{*}+u_{4}(t)\right),
$$

where $x^{2}=\left(x_{3}, x_{4}\right)$ and $\left|u_{4}(t)\right| \leq u_{4}^{*}$. The latter is again a requirement in order to $u_{4}^{*}+u_{4}(t)$ represents only "introduction" of larvae parasitoids.

Now, to $u_{4}^{*}$ defined in (3.6) and $u_{4}(t):=0 \quad(t \in[0, T])$, there corresponds the desired positive equilibrium $\left(x_{d 1}^{*}, x_{d}^{2^{*}}:=\left(x_{d 3}^{*}, x_{d 4}^{*}\right)\right)$.

For local controllability of control system (3.11) to $\left(x_{d 1}^{*}, x_{d}^{2^{*}}\right)$, on $[0, T]$, we calculate the linearization

$$
\begin{gathered}
A_{11}:=\frac{\partial F_{1}}{\partial x^{2}}\left(x_{d 1}^{*}, x_{d}^{2^{*}}, u_{4}^{*}\right)=\left[\begin{array}{cc}
-m_{3}-n_{3}-\beta x_{d 4}^{*} & -\beta x_{d 3}^{*} \\
\beta \gamma_{2} x_{d 4}^{*} & 0
\end{array}\right], \\
A_{10}:=\frac{\partial F_{1}}{\partial x_{1}}\left(x_{d 1}^{*}, x_{d}^{2^{*}}, u_{4}^{*}\right)=\left[\begin{array}{c}
n_{1} \\
0
\end{array}\right] ; B_{1}:=\frac{\partial F_{1}}{\partial u_{4}}\left(x_{d 1}^{*}, x_{d}^{2^{*}}, u_{4}^{*}\right)=\left[\begin{array}{l}
0 \\
1
\end{array}\right] .
\end{gathered}
$$

From

$$
\operatorname{det}\left[B_{1} \mid A_{11} B_{1}\right]=\beta x_{d 3}^{*} \neq 0,
$$

again we get $\operatorname{rank}\left[B_{1} \mid A_{11} B_{1}\right]=2$, and applying sufficient condition (1.3), we obtain the local controllability of system (3.11) into $x_{d}^{2^{*}}$, on interval $[0, T]$. Hence, by the analogous rank conditions for $\left(L V_{i}\right)$ recalled in subsection "Verticum-structured model", applying Theorem 1 we easily obtain the following results:

\section{Theorem 2}

A) Under conditions (3.8), control system (3.9) is locally controllable into its equilibrium $x_{d}^{*}$.

B) If $u^{*}:=0$, and the parameters of system (3.1) satisfy conditions (3.3), then system (3.4) is locally controllable to the original equilibrium $x^{*}$, on interval $[0, T]$. 


\section{Optimal control problem}

3 The problem is to control system (3.9) from state $x(0)=x^{0}$ to the new desired equilibrium $x_{d}^{*}$ 4 in $[0, T]$. Controllability obtained in part A) of Theorem 2, in principle guarantees the existence 5 of such control. We can concretely calculate such control by solving following optimal control 6 problem:

$$
\begin{gathered}
\Psi(u):=\left|x(T)-x_{d}^{*}\right|^{2} \rightarrow \min , \\
u \in U_{\varepsilon}[0, T], \\
x^{\prime}=F\left(x_{1}, x_{2}, x_{3}, x_{4}, u^{*}+u\right), \\
x(0)=x^{0}, \quad x(T)=x_{d}^{*} .
\end{gathered}
$$

For the solution, the toolbox developed for MatLab in Banga et al. (2005) and Hirmajer et al. (2009) is applied. In the numerical approximation, piece-wise constant functions (step functions) are used as controls.

We note that in the biocontrol practice agent releases usually occur in pulses, but a constant rate release can be well approximated with an appropriate technique. (See Driesche and Bellows, (2001) for parasitoid agents, or Vila and Cabello, (2014) for predator agents, and Shi et al., (1988); Knutson, (1998) for parasitoid species of the Trichogramma genus.) The technique in question consists in placing dispensers in the field (say once a week), containing agents of different development stages that develop to adult age (and leave the dispenser) gradually, which results in a release very close to a constant daily release.

Example. For our model calculations we adapt the same parameters of Rafikov and Silveira (2013) originally obtained from field experiments (see Parra et al. (2002), and for the methodology of the necessary trials we refer to Ambrosano et al., (1996)):

$$
\begin{aligned}
& r=0.19 ; K=25000 ; m_{1}=0.03566 ; m_{2}=0.03566 ; m_{3}=0.00256 ; m_{4}=1 ; n_{1}=0.1 ; \\
& n_{3}=0.02439 ; \alpha=0.0000075 ; \beta=0.0000083 ; \gamma_{1}=2.29 ; \gamma_{2}=40 .
\end{aligned}
$$

Then conditions (3.3) are fulfilled, implying a stable coexistence of the population. For these parameters, we have the following positive equilibrium of model (3.1):

$$
x_{1}^{*}=2076.27 ; x_{2}^{*}=5141.38 ; x_{3}^{*}=3012.05 ; x_{4}^{*}=5058.11 \text {. }
$$

For system (3.1), with initial condition $x^{0}:=(1000,7000,2500,2000)$, the corresponding solution $x$, tending to equilibrium $x^{*}=\left(x_{1}^{*}, x_{2}^{*}, x_{3}^{*}, x_{4}^{*}\right)$, can be seen in Figure 1. 


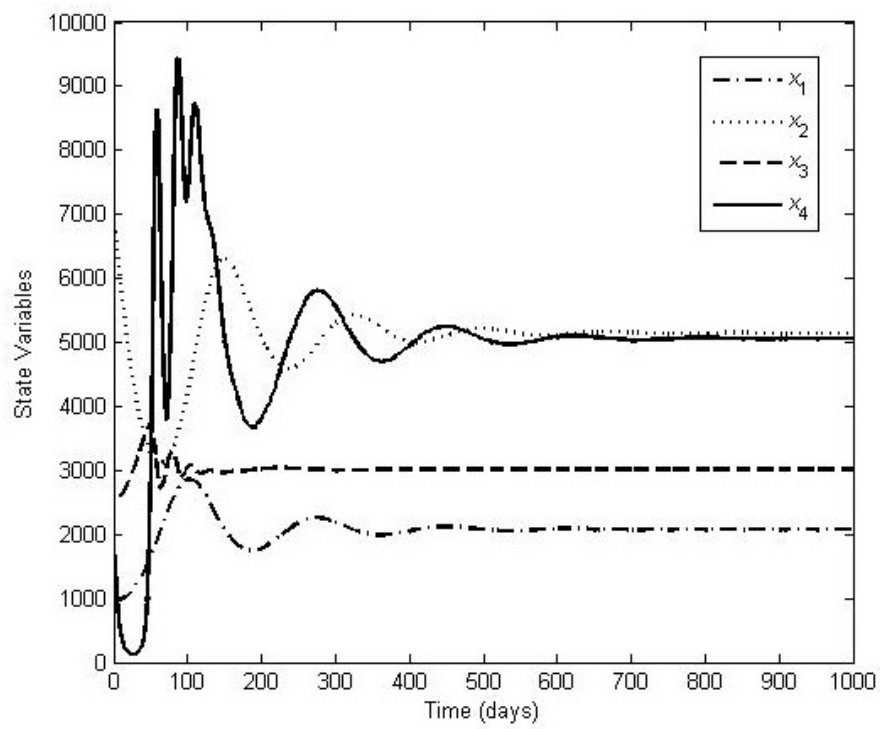

Figure 1. Solution of system (3.1), with initial value $x^{0}:=(1000,7000,2500,2000)$

Advantages of the verticum approach

We want to steer the state of the pest larvae population to a new desired equilibrium value, lower than the previous one (higher than 3000). Let us suppose that the crop suffers important damages if exposed to pest larvae levels higher than 2100 during a long period of time. Lower pest levels does not injure the crop. Therefore, our aim will be to reduce the pest larvae equilibrium to a value lower than the critical value 2100 , say $x_{d 3}^{*}=2000$. Then we fixed the desired value of the pest eggs density, for instance, at $x_{d 1}^{*}=800$, obtaining the desired new equilibrium state from (3.7):

$$
x_{d}^{*}=\left(x_{d 1}^{*}, x_{d 2}^{*}, x_{d 3}^{*}, x_{d 4}^{*}\right)=(800,6434.67,2000,1572.29),
$$

and the constant control from (3.6):

$$
u^{*}=\left(u_{2}^{*}, u_{4}^{*}\right)=(141.048,528.289) .
$$

For these parameters, condition (3.8) is satisfied, thus $x_{d}^{*}$ is a positive asymptotically stable equilibrium of system (3.10)-(3.11).

Applying the previous results, our objective is to determine a control of system (3.10)-(3.11), that steers the state into this new equilibrium.

As an illustrative value for the length of the total growing period, let us fix time duration $T:=200$ (days) and take initial condition $x^{0}:=(1000,7000,2500,2000)$. For the calculation of the corresponding solution of the optimal control problem (3.12) we apply the toolbox developed for MatLab in Banga et al. (2005) and Hirmajer et al. (2009).

We will see that, a) if we solve (3.10)-(3.11) by subsystems (in other words by verticum approach), that is, by solving the optimal control problem first for subsystem (3.10) and then for subsystem (3.11) with $x_{1}:=x_{d 1}^{*}$ calculated for the previous subsystem (see Figure 2.a)), in several aspects we obtain better result than b) solving the optimal control problem without decomposition (see Figure 2.b)).

Indeed, in Figure 2, observe that in case a) pest larval density decreases below the threshold value much quicker (in time $T_{1}$ ) than in case b) (in time $T_{2}$ ). During time $T_{2}-T_{1}$ the pest 
larvae may cause a serious damage. If this damage is fatal, the only option is the verticum approach a). So the time of crossing the critical level is good indicator of which method is better. Below we shall also see that the verticum approach may be better even in terms of cost effectiveness.

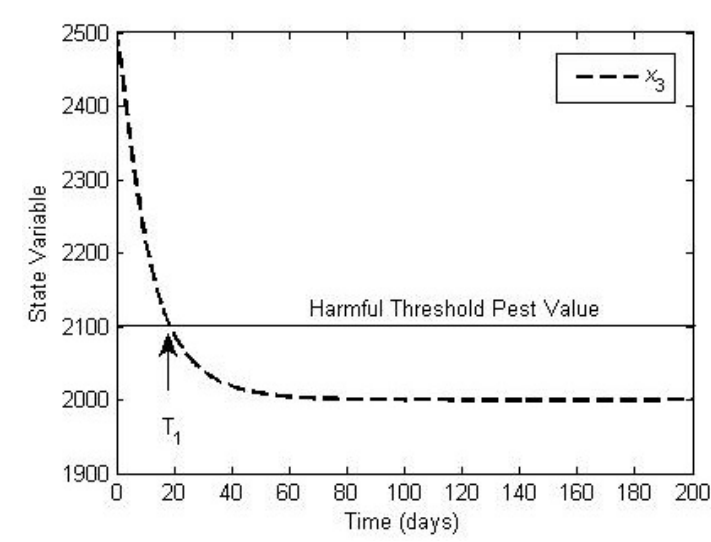

a)

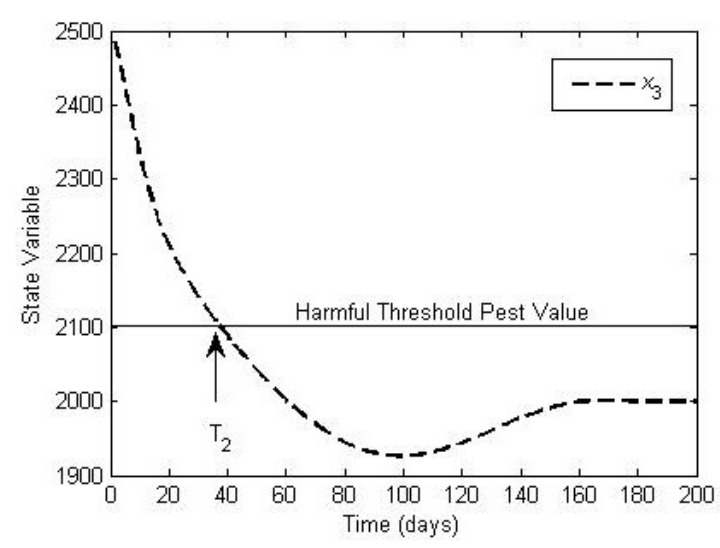

b)

Figure 2. a) Solution of control system (3.10)-(3.11) by subsystems (with $x_{1}=800$ in the second one) for $T=200$ with initial values $x^{01}:=(1000,7000)$ and $x^{02}:=(2500,2000)$. b) Solution of control system (3.10)-(3.11) without decomposition for $T=200$, with initial value $x^{0}:=(1000,7000,2500,2000)$.

Let $p_{1}$ and $p_{2}$ be weights expressing the proportions of the costs of production and release of both agents. Then the total cost corresponding to control functions $u_{2}$ and $u_{4}$ is

$$
C\left(u_{2}, u_{4}\right):=\int_{0}^{T}\left(p_{1} \cdot\left[u_{2}^{*}+u_{2}(t)\right]+p_{2} \cdot\left[u_{4}^{*}+u_{4}(t)\right]\right) d t
$$

For an illustration, set $p_{1}=0.13, p_{2}=0.87$ (T. Cabello, 2014, Com. Pers.). Then, if we solve the optimal control problem (3.12) for control system (3.10)-(3.11), for $T=200$, without decomposition, with initial value $x^{0}:=(1000,7000,2500,2000)$, then the total cost expressed in (3.15) is $9.558 \cdot 10^{4}$. If we solve the same optimal control problem for control system (3.10)-(3.11) with the verticum approach (i.e. by subsystems), with initial values $x^{01}:=(1000,7000)$ and $x^{02}:=(2500,2000)$, the total cost is $9.561 \cdot 10^{4}$ which is only $0.03 \%$ more than in the case of optimization without decomposition. However, as we have seen before, the verticum type optimal control performs much better in controlling the pest larval density below the critical threshold in half the time. In this sense the verticum approach turns out to be more cost effective. Therefore, in what follows we will solve the considered optimal control problems applying the verticum approach.

Remark 4. The particular weighting of the costs of parasitoid control (with $p_{1}=0.13$ ) comes from the biocontrol practice. Now, for an outlook, we also make simulations with different proportions $p_{1}: p_{2}$, letting $p_{1}$ run from 0.2 to 0.8 . As we see from Table 1 , the additional cost of the verticum approach remains below $1 \%$, which also suggests that the verticum approach may also be useful in the context of other two-agent biocontrol plans.

In any case, if the biocontrol system is of verticum type, it is worth it to make simulations with both methods (verticum and non-verticum approach) to see which one performs better. 


\begin{tabular}{|c|c|c|c|c|}
\hline$p_{1}$ & $p_{2}$ & $\begin{array}{c}\text { Total Cost (without } \\
\text { decomposition) }\end{array}$ & $\begin{array}{c}\text { Total Cost (by } \\
\text { verticum structure) }\end{array}$ & difference (\%) \\
\hline 0.2 & 0.8 & $9.015 \cdot 10^{4}$ & $9.02 \cdot 10^{4}$ & $0.05 \%$ \\
\hline 0.4 & 0.6 & $7.421 \cdot 10^{4}$ & $7.475 \cdot 10^{4}$ & $0.7 \%$ \\
\hline 0.6 & 0.4 & $5.917 \cdot 10^{4}$ & $5.924 \cdot 10^{4}$ & $0.1 \%$ \\
\hline 0.8 & 0.2 & $4.352 \cdot 10^{4}$ & $4.377 \cdot 10^{4}$ & $0.05 \%$ \\
\hline
\end{tabular}

4

Table 1. Cost-effectiveness of the verticum approach for different proprtions between the agent costs

$$
\text { difference }(\%)=\frac{\text { Total Cost }(\text { by verticum structure })-\text { Total Cost }(\text { without decomposition })}{\text { Total Cost }(\text { without decomposition })} \cdot 100
$$

In Figure 3a) we can see a comparison between the pest larval density in the uncontrolled system (3.1) and in the equilibrium controlled system (3.10)-(3.11), calculated by subsystems. It is easy to judge the effect of an appropriate biological control: without applying any control, the pest larval density is all the time above the harmful level and displays many abrupt fluctuations, while applying the equilibrium control, the larvae trajectory is much more soft and appropriate, and remains below the critical level.

Figure 3b) shows all coordinates of the solution of the control system (3.10)-(3.11) by subsystems (with $x_{1}=800$ in the second one) with initial values $x^{01}:=(1000,7000)$ and $x^{02}:=(2500,2000)$, ending up at desired equilibrium $x_{d}^{*}=(800,6434.67,2000,1572.29)$. Moreover, as we can observe, the solution of the control system arrives at the desired equilibrium way before the solution of the uncontrolled system reaches its own equilibrium. The obtained optimal control $u=\left(u_{2}, u_{4}\right)$ can be seen in Figure 3c).

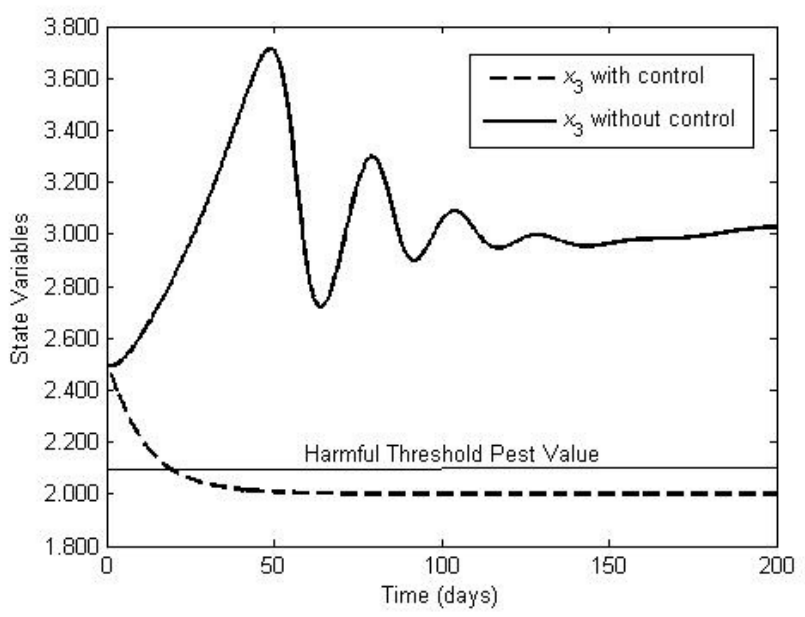

a) 


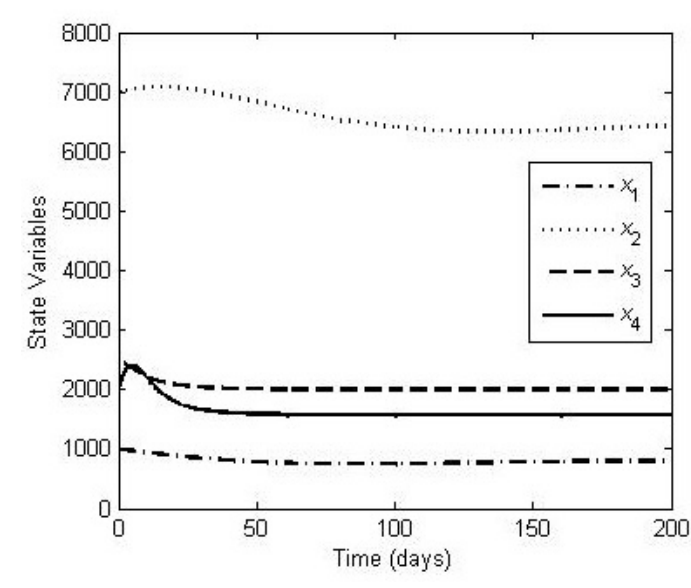

b)

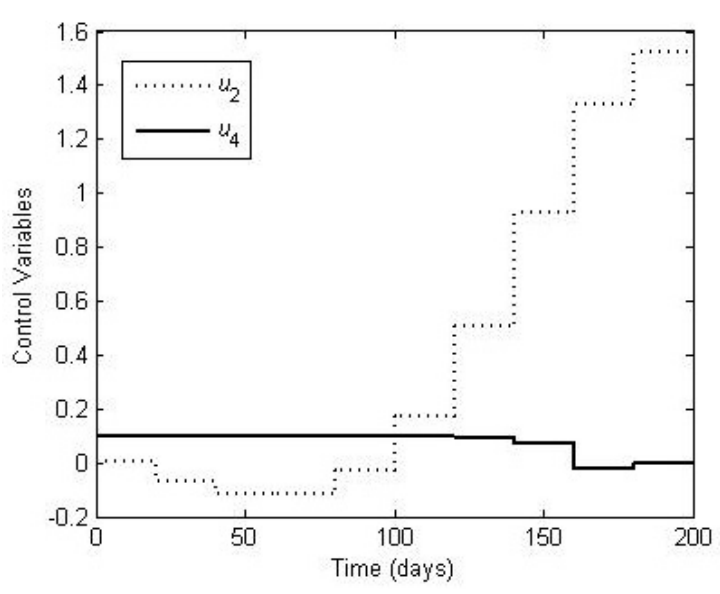

c)

Figure 3. a) Comparison of the third coordinate (the pest larval density) of the solution of the uncontrolled system (3.1) with initial value $x^{0}:=(1000,7000,2500,2000)$ and the third coordinate of the solution of the optimal control problem for control system (3.10)-(3.11) by subsystems (with $x_{1}=800$ in the second one) for $T:=200$, with initial values $x^{01}:=(1000,7000)$ and $x^{02}:=(2500,2000)$, b) Solution of the optimal control problem for control system (3.10)-(3.11) by subsystems for $T:=200$, with initial values $x^{01}:=(1000,7000)$ and $x^{02}:=(2500,2000)$,

c) Optimal control function for system (3.10)-(3.11) solved by subsystems for $T:=200$.

\section{Controlling to a new equilibrium along a given trajectory}

Until now our aim has been to drive the pest larval density to a new, more appropriate equilibrium in a given time $T$. At this point we add a further requirement of steering the pest larval density $x_{3}$ to this equilibrium along a given curve $\varphi(t)$, with $\varphi(0)=x_{3}(0)$ $>\varphi(T)=x_{d 3}^{*}$. We may require e.g. that a) at a lower cost, we start with a softer decrease of $x_{3}$, continuing with a stronger decrease (concave parabola); b) the rate of decrease of $x_{3}$ is uniform (following a straight line); c) we start with a stronger decrease, continuing with a softer decrease of $x_{3}$ (convex parabola): For $t \in[0, T]$ let

a) $\varphi(t):=a x^{2}+c, \quad$ with $a:=\frac{x_{d 3}^{*}-x_{3}(0)}{T^{2}}$ and $c:=x_{3}(0)$

b) $\varphi(t):=a x+b, \quad$ with $a:=\frac{x_{d 3}^{*}-x_{3}(0)}{T}$ and $b:=x_{3}(0)$

c) $\varphi(t):=a x^{2}+b x+c, \quad$ with $a:=\frac{x_{3}(0)-x_{d 3}^{*}}{T^{2}}, b:=-2 a T$ and $c:=x_{3}(0)$

\section{Optimal control problem}

The problem is to control system (3.10)-(3.11) by subsystems from state $x(0)=x^{0}$ to the new desired equilibrium $x_{d}^{*}$ in $[0, T]$, moving $x_{3}(t)$ along the given curve $\varphi(t)$. Then we have to solve the following optimal control problem:

$$
\text { With some } q_{1}, q_{2} \geq 0, \quad q_{1}+q_{2}=1,
$$




$$
\Psi(u):=q_{1}\left|x(T)-x_{d}^{*}\right|^{2}+q_{2} \int_{0}^{T}\left|x_{3}(t)-\varphi(t)\right|^{2} d t \rightarrow \min
$$

$$
u \in U_{\varepsilon}[0, T]
$$

$$
x^{\prime}=F\left(x_{1}, x_{2}, x_{3}, x_{4}, u^{*}+u\right)
$$

$$
x(0)=x^{0}, x(T)=x_{d}^{*}
$$

For the solution, again the toolbox developed for MatLab in Banga et al. (2005) and Hirmajer et al. (2009) is applied. For the following numerical examples we shall use illustrative values $7 \quad q_{1}=0.6, q_{2}=0.4$.

A comparison between the pest larval density and the three prescribed trajectories is shown in Figure 4. We can say that the approximation to the given functions is quite acceptable. Although the rest of the coordinates are not shown, they also arrive at the corresponding equilibrium 11 values.

12

13

14

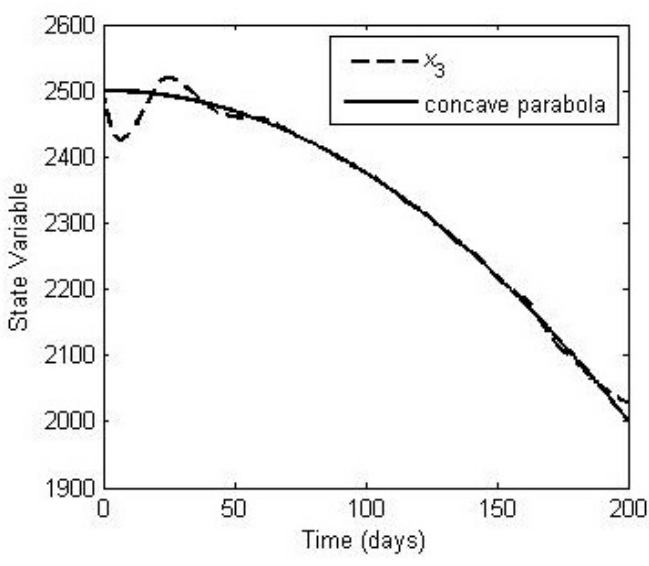

a)

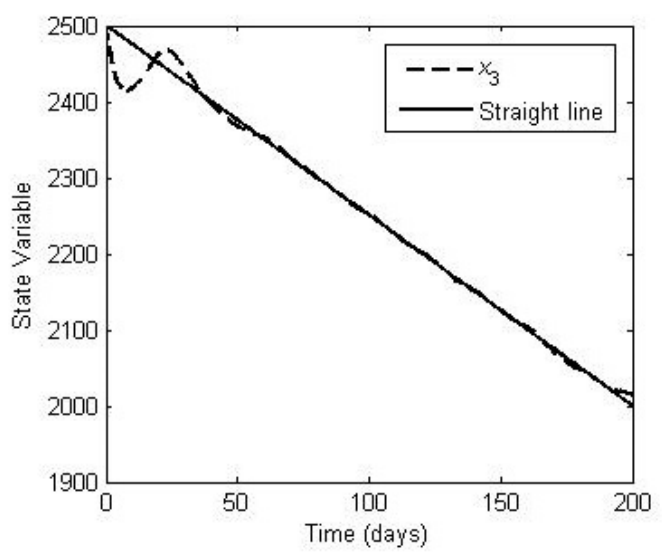

b)

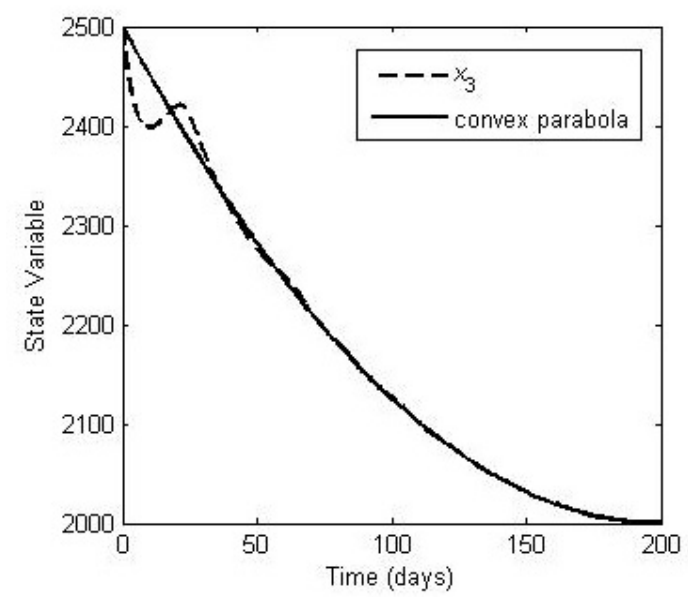

c)

Figure 4. a) Function $\varphi$ given in (3.16) and pest larval density approximating it, resulting from the optimal control problem (3.19) with dynamics (3.10)-(3.11), solved by subsystems for $T=200$, with initial values $x^{01}:=(1000,7000)$ and $x^{02}:=(2500,2000)$. In b) and c) similar approximations are shown for functions $\varphi$, given in (3.17) and (3.18), respectively. 
The corresponding total costs of controlling the population to the new equilibrium along these three trajectories are: in case a) $4.678 \cdot 10^{4}$, in case b) $5.799 \cdot 10^{4}$, in case c) $7.172 \cdot 10^{4}$.

As we can see, when we steer the pest larval density along a concave curve, the cost is less than in the other two cases. It is intuitively clear that, the larger the area under the curve is, the greater is the damage caused by the larvae. Therefore, the presented methodology is an efficient tool for the "in silico" analysis of the cost-effectiveness of different biocontrol strategies.

\section{Controlling to the new equilibrium in less time}

Besides driving the population system into a new desired equilibrium, it could be also convenient to arrive at this state in less time. Under the condition that the crop duration is longer than $T=200$ days, in the previous subsection we have shown how to control the system to the new equilibrium in this time. Now we are going to see that it is also possible to reach this equilibrium in only 20 days. The question is if it is economically worthwhile. Let us check it. Firstly, in Figure 5a) we can see that at time $T=20$ the population ends up in the new equilibrium, Figure $5 \mathrm{~b}$ ) shows the optimal control $u=\left(u_{2}, u_{4}\right)$ realizing this.

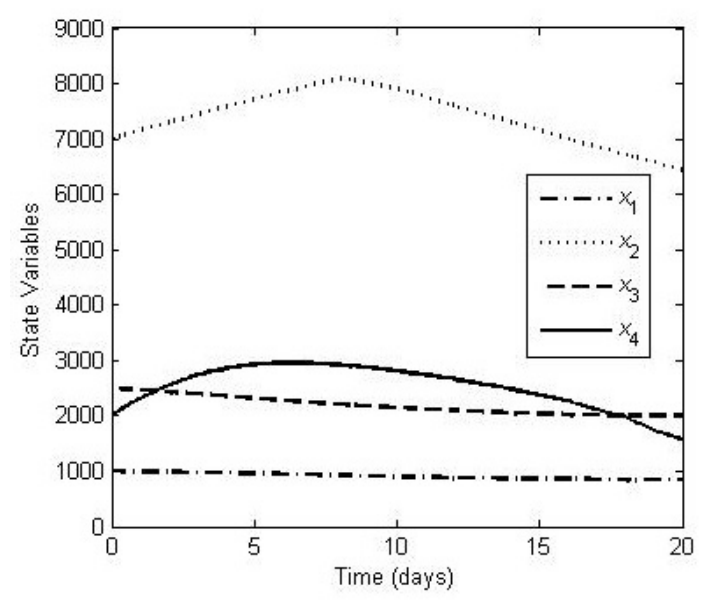

a)

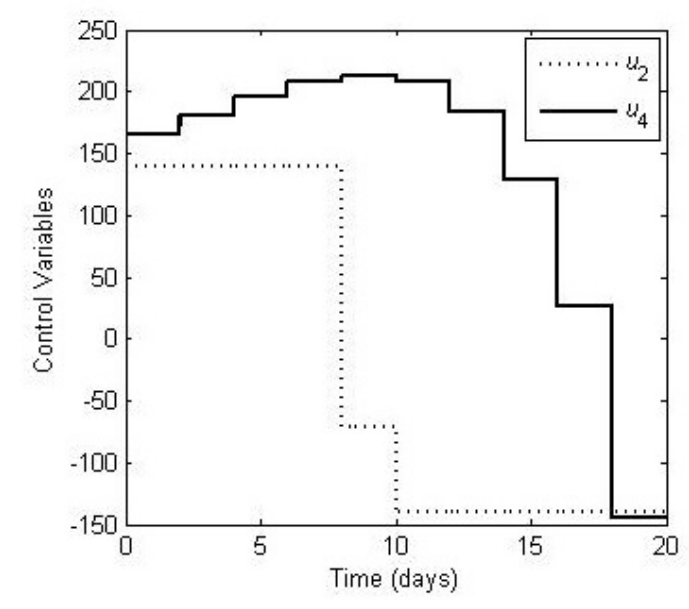

b)

Figure 5. a) Solution of system (3.10)-(3.11) the controlled by subsystems for $T=20$, with initial values $x^{01}:=(1000,7000)$ and $x^{02}:=(2500,2000)$. b) Control function of system (3.10)-(3.11) obtained by subsystems for $T=20$.

Secondly, if the crop season is, say, 200 days, in order to keep this equilibrium state of the population system for the remaining 180 days, it is necessary to maintain the constant control $u^{*}=\left(u_{2}^{*}, u_{4}^{*}\right)$ during the last 180 days. Now we calculate the costs of both options:

Total cost of controlling to the equilibrium in $T=200$ days is $9.561 \cdot 10^{4}$ (calculated in Section 3.1).

Total cost of controlling to the equilibrium by $T=20$ and maintaining the system in this state until $T=200$ :

$$
\begin{aligned}
& \int_{0}^{20}\left(0.13 \cdot\left[u_{2}^{*}+u_{2}(t)\right]+0.87 \cdot\left[u_{4}^{*}+u_{4}(t)\right]\right) d t+\int_{20}^{200}\left(0.13 \cdot\left[u_{2}^{*}+0\right]+0.87 \cdot\left[u_{4}^{*}+0\right]\right) d t= \\
& \quad=97910.586
\end{aligned}
$$

The difference between both costs is around $2.35 \%$. Thus, at a barely higher cost, the system can be controlled quickly to the desired equilibrium and then kept there, such that the crop is exposed to a harmful pest larvae level less time than with the long term equilibrium control. 
This example illustrates that our modelling approach and simulation analysis can contribute to the improvement of the cost effectiveness of the applied biocontrol strategy.

\section{CONCLUSIONS}

5 The ecological basis of biological pest control is the interaction between a pest population and 6 its natural enemies (predators or parasitoids). Based on appropriate population dynamics 7 models, for the determination of an appropriate control strategy, optimal control theory (or more 8 generally, mathematical systems theory) turned out to be an adequate tool.

9 In Rafikov et al. (2008), optimal control already was applied to biocontrol of sugar cane borer the two- agent model of Rafikov and Silveira (2013). While in the latter optimal asymptotic feedback control is obtained, our results concern the control of the population into a desired equilibrium in given time. In an earlier paper (Molnár et al., 2013) the foundations of nonlinear verticum type control systems were laid down, and applied to integrated pest control of the sugar cane borer, based on the single-agent biocontrol model of Rafikov et al. (2008).

In the present work instead, in the context of a four-dimensional, stage-structured two-agent biocontrol model, we gave an insight into the advantages of the application of a verticum (or decomposition) approach to biological control, analyzing the effectiveness of this control methodology.

For each subsystem of the verticum-type population system, we have obtained the corresponding control function to steer the state population of each subsystem to its corresponding new desired equilibrium in a given time, providing an equilibrium for the whole system, where the pest larval density is below a critical threshold. Our model also makes it possible to calculate the cost of such biocontrol strategy, providing in this way an efficient pest management approach to avoid serious economic damages in terms of crop quality and/or quantity.

With our model we can also analyze the effect of steering the population system to the new desired equilibrium along a partially prescribed trajectory according to the convenience of the situation. We note that this is different from the classical trajectory tracking problem of systems theory, since here we prescribe only one coordinate (pest larval density) of the time-dependent state vector). For different partially prescribed trajectories we have compared the total cost of the corresponding control strategies in our illustrative example.

This decomposition type control methodology is also promising in the equilibrium control of large ecosystems. In fact, its methodological advantage may be that the analytical study of controllability is technically simpler with the decomposition approach, and hence the biological interpretation of the sufficient condition for controllability may be substantially easier.

Analogously to the technique used in this paper, we could have obtained the corresponding controls to steer the population to the equilibrium state not only applying different types of biological control (involving e.g. autochthonous predators), but also using integrated pest control, combining the initial short-term effect of a chemical control with the softer effect of biological control, allowing sufficient time for both the biological agent to establish and the pesticide to decompose before the harvest.

Finally, for biocontrol technicians we shortly summarize the motivation and applicability of the optimal control methodology in biological pest control practice, where the applied control function is always a release of two possible agents: egg and larvae parasitoids. A general objective is to control and keep the pest larvae density below an "economic threshold". The latter concept was first introduced for chemical pest control (see e.g. Dent, 2000), and means the 
insect's population level at which the value of the crop destroyed exceeds the cost of controlling the pest, which was easily adapted to the case of two-agent biological control, too.

The development of our optimal control model for the two-agent case is justified by the fact that none of the considered parasitoids alone can control the considered pest (see e.g. Driesche et al., 2008).

We suggested four possible field applications:

a) Controlling the three species population system in given time into a required equilibrium, where the target density of the pest larvae is below a given critical threshold value. (Actually, the control is realized by minimizing the distance from the prescribed equilibrium.)

b) Comparing the costs of the above equilibrium control with and without the decomposition method (controlling the host-egg parasitoid system and host-larvae parasitoid systems separately, or together), in order to see which one performs better and at what cost.

c) The time of reaching the required equilibrium (where density of the pest larvae is low enough) can be shortened, and its additional cost can be also calculated for a reasonable decision.

d) The decrease of the pest larvae density can be achieved along different trajectories, at different costs. The presented optimal control method can also provide the control strategy that realizes a given trajectory, also indicating the corresponding costs, supporting the biocontrol technician in finding cost-effective agent release strategies.

\section{ACKNOWLEDGEMENTS}

Research was supported by the Hungarian National Scientific Research Fund OTKA (K81279) and also funded by the Regional Government of Andalusia (Spain), Programme of Excellence Project (ref: P11-TIC-7821) of the Junta de Andalusia, Consejeria de Economia, Innovacion y Ciencia, with joint financing from FEDER Funds. The authors acknowledge the anonymous reviewers as well as Prof. Tomás Cabello and Prof. Marat Rafikov for their suggestions to improve the paper.

\section{REFERENCES}

Ambrosano, G. M. B., Stimac, J. L., Neto, S. S., Igue, T., Nagai, V. "Modelo matemático para simulação do controle biológico da broca-da-cana com o parasitóide Trichogramma galloi: Modelos Conceituais". XII. Metodologia e Técnicas Experimentais, Bragantina, Campinas, 1996. [7] Bassanezi, R.

Banga, J.R., E. Balsa-Canto, C.G. Moles and A.A. Alonso (2005), Dynamic Optimization of Bioprocesses: Efficient and Robust Numerical Strategies, Journal of Biothecnology, Vol. 117, pp. 407-419.

Dent, D. (2000). Insect pest management. 2nd Edition. CABI Publishing. Wallingford, UK: 410 pp.

Driesche, R.G. van; Bellows, T.S. (2001). Biological control. Third printing. Kluwer Academic Publishers. Dordrecht, NL: 539 pp. 
Driesche, R. van; Hoddle, M.; Center, T. (2008). Control of pests and weeds by natural enemies: An introduction to biological control. Blackwell Publishing, Malden, MA: 473 pp.

Gámez, M., López, I., Garay, J. and Varga, Z. (2009). Observation and control in a model of a cell population affected by radiation. Biosystems 96 (2009) 172-177.

6 Gámez, M. López, I., Szabó, I. and Varga, Z. (2010). Verticum-type systems applied to 7 ecological monitoring. Applied Mathematics and Computation, 215, 3230-3238.

8 M. Gámez, "Observation and Control in Density- and Frequency-dependent Population 9 Models," In: Zhang (Ed.), Ecological Modeling, Nova Science Publish- ers, New York, 2011, 10 pp. 285-306.

11 Gámez, M., López, I., Garay, J. and Varga, Z. (2011). Monitoring and control in a spatially Apduhan (Eds.), Computational Science and Its Applications Vol. V pp. 511-520, "Lecture Notes in Computer Science" 6786, Springer-Verlag Berlin Heidelberg.

Gámez, M., López, I., Varga Z. and Garay, J. (2012). Stock estimation, environmental monitoring and equilibrium control of a fish population with reserve area. Reviews in fish biology and fisheries, 22 (3), 751-766

Hirmajer, T., E. Balsa-Canto and J. Banga (2009), DOTcvpSB, a Software Toolbox for Dynamic Optimization in Systems Biology, BMC Bioinformatics, Vol. 10, pp. 199.

Knutson, A. (1998). The Trichogramma manual. Texas Agricultural Extension Service, The Texas A\&M University System: 42 pp.

[http://university.uog.edu/cals/people/pubs/insbene/b6071.pdf]

Lee, E.B.; Markus, L. (1971). Foundations of Optimal Control Theory. Wiley, New YorkLondon-Sydney

López, I., Gámez, M. and Carreño, R. (2004). Observability in dynamic evolutionary models. Biosystems, 73, 99-109.

López I, Gámez M, Molnár, S. (2007a). Observability and observers in a food web. Applied Mathematics Letters 20 (8): 951-957.

López, I., Gámez, M., Garay, J. and Varga, Z. (2007b). Monitoring in a Lotka-Volterra model. BioSystems Vol. 87, No. 1, 68-74.

Macedo, N. \& P.S.M. Botelho. (1988). Controle integrado da broca da cana-de-açúcar, Diatraea saccharalis (Fabr., 1794) (Lepidoptera: Pyralidae). Brasil Açucareiro 160: 2-14.

Molnár, S. (1989). A special decomposition of linear systems. Belgian Journal of Operations Research, Statistics and Computer Science, 29 (4): 1-19.

Molnár, S. (1993). Stabilization of verticum-type systems, Pure Mathematics and Applications, 4 (4): 493-499.

Molnár, S., Gámez, M. and López, I. (2012). Observation of nonlinear verticum-type systems applied to ecological monitoring. International Journal of Biomathematics. DOI No: 10.1142/S1793524512500519

Molnár, S., Gámez, M., López, I. and Cabello, T. (2013). Equilibrium control of nonlinear verticum-type systems, applied to integrated pest control. Biosystems 13: 72-80.

Molnár, S., Szigeti, F. (1994). On "Verticum"-Type Linear Systems with Time-Dependent Linkage. Applied Mathematics and Computation 60: 89-102.

Parra, J.R.P., Botelho, P.S.M., Ferreira, B.S.C., Bento, J.M.S., 2002. Controle Biológico no Brasil: Parasitóides e Predadores. Editora Manole, Sao Paulo. 
Rafikov M, Balthazar JM, von Bremen HF (2008) Mathematical modelling and control of population systems: applications in biological pest control. Appl. Math. Comput. 2:557-573

Rafikov, M., Limeira, E.H. (2012). Mathematical Modelling of the Biological Pest Control of the Sugarcane Borer, International Journal of Computer Mathematics, Vol 89, No 3, 390-401.

6 Rafikov, M., \& Silveira, J. C. (2013). Dynamics of the sugarcane borer-egg parasitoid-larvae 7 parasitoid agro-ecosystem. International Conference MPDE'13-Models in Population Dynamics and Ecology. University of Osnabrück, Germany, 26-29 August, 2013. Abstracts, p. 57

Rafikov, M. Bevilacqua, L., Wyse, A.P.P. (2009), Optimal control strategy of malaria vector June, Pages 418-425.

Rafikov Marat, Silveira, Jean Carlos (2014), On dynamical behavior of the sugarcane borerparasitoid agroecosystem, Ecological Complexity, Volume 18, June 2014, Pages 67-73.

Scarelli, A., Varga, Z. (2002). Controllability of selection-mutation systems. BioSystems. 65, No 2-3, 113-121.

Shamandy, A. (2005). Monitoring of trophic chains. Biosystems, Vol. 81, No. 1, 43-48.

Shi, G.Z.; Zhou, Y.N.; Zhao, J,S.; Li, T.; Lian, M.L.; Chang, R.Q.; Li, T.X.; Chen, S.; Yang, X.L.; Niou, J.Q. (1988). The techniques of protection of the Trichogramma population in the fields. Colloques de I'INRA, 43. INRA Publ., Paris, FR: 581-583 pp.

Varga, Z. (1989). On controllability of Fisher's model of selection. In "Differential Equations" (Eds. C. M. Dafermos, G. Ladas, G. Papanicolau) Marcel Dekker, New York, 717-723.

Varga, Z. (1992). On Observability of Fisher's model of selection. Pure Mathematics and Applications, Ser. B. Vol. 3 No 1, 15-25.

Varga, Z., Scarelli, A. and Shamandy, A. (2002). An observability problem of population ecology. In: V. Capasso (Ed.), Mathematical Modelling and Computing in Biology and Medicine, 5th ECMTB Conference 2002 (Abstracts, p.189). MIRIAM, Milan, Italy.

Varga, Z., Scarelli, A., Shamandy, A. (2003). State monitoring of a population system in changing environment. Community Ecology, 4, (1) 73-78.

Varga, Z. (2008a). Observer Design for Genetic Processes. Mechanical Engineering Letters Vol. 1, 13-25.

Varga, Z. (2008b). Applications of mathematical systems theory in population biology. Periodica Mathematica Hungarica, Vol. 56 (1), 157-168.

Z. Varga, M. Gámez, I. López (2013) Recent developments in monitoring of complex population systems. American Journal of Operations Research, 2013, 3, Number 1 (Special issue on Complex Systems) 167-180.

Vila, E.;Cabello, T. (2014). Biosystems engineering applied to greenhouse pest control, In Torres, I. and Guevara, R. (eds). Biosystems engineering: Biofactories for food production in the XXI Century. Springer, Berlin, DE: pp 99-128. 